\title{
Algunos musgos de Tabasco, México
}

\author{
Claudio Delgadillo M. ${ }^{1}$ y Sergio Zamudio ${ }^{2}$
}

\begin{abstract}
RESUMEN. Aunque la flora de musgos del estado de Tabasco contiene, por inferencia, numerosos taxa, hasta ahora sólo se habían registrado seis. Los registros de la presente contribución elevan el número conocido a 47 especies y variedades, pero nueve de ellos provienen del límite con el estado de Chiapas.
\end{abstract}

ABSTRACT. Although it may be inferred that the moss flora of the Mexican state of Tabasco contains numerous taxa, to date only six have been recorded. In this contribution, new records for the moss flora of Tabasco bring up the number to 47 species and varieties; nine of them, however, were obtained from the borderline with Chiapas.

Hay varios estados de la República Mexicana en los que la exploración briológica ha sido notablemente exigua. Tabasco, entre ellos, no ha recibido mayor atención posiblemente por razones geográficas e históricas. En comparación con Veracruz, Tabasco no cuenta con elevaciones significativas y no ha estado dentro de las rutas comerciales que han propiciado la recolección asidua de plantas.

Además del desconocimiento briológico, la ganadería y la explotación petrolera han alterado significativamente el paisaje y muchas áreas potencialmente importantes no han dejado huella de su valor florístico. La exploración actual, sin embargo, puede rescatar información que nos permite evaluar el significado florístico y fitogeográfico del estado con respecto a otras zonas de la vertiente oriental de México y de América Central.

En 1983 uno de los autores (Sergio Zamudio) inició la recolección de musgos de Tabasco en conjunción con otros individuos y en relación con trabajos florísticos. La colección es pequeña e incluye muchas especies que son relativamente frecuentes en las zonas tropicales de México; hasta ahora no se ha hecho una colección exhaustiva en cada sitio muestreado sino que se han visitado zonas muy diversas (fig. 1) por lo cual el listado no representa un perfil de la flora de musgos de Tabasco.

${ }^{1}$ Departamento de Botánica, Instituto de Biología, Universidad Nacional Autónoma de México, Apdo. Postal 70-233; 04510, México, D.F.

${ }^{2}$ Centro de Investigación y Desarrollo del Estado de Michoacári, Apdo. Postal 386; 61600 Pátzcuaro, Mich., México. 


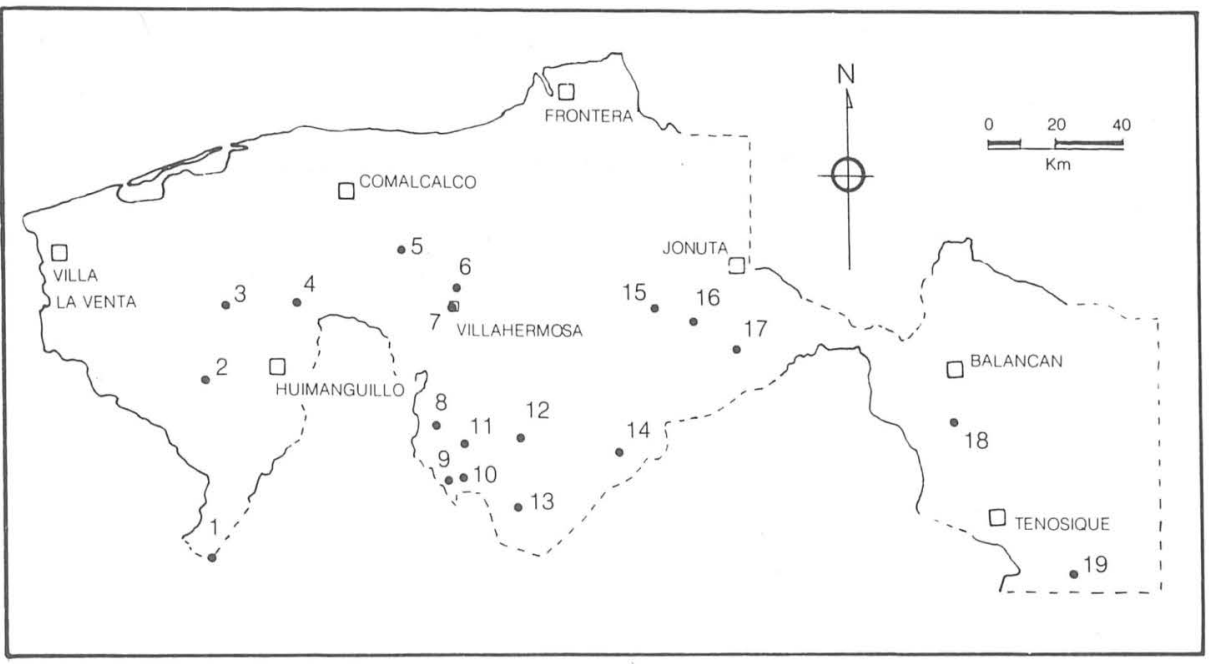

Fig. 1. Localidades de colecta de musgos de Tabasco. Los números en el mapa representan las comunidades vegetales descritas en el texto.

En los párrafos que siguen se enumeran las localidades (cf. fig. 1) con los números de colecta de los ejemplares y algunos datos de la vegetación de cada zona. En todos los casos la numeración corresponde a la de Sergio Zamudio. En la lista se incluyen ejemplares provenientes del cerro Mono Pelón (Localidad Núm. 1) situado en los límites de Veracruz y Chiapas; las etiquetas de herbario los sitúan dentro de Chiapas pero desde un punto de vista florístico, también pueden atribuirse a Tabasco. Tales ejemplares se señalan con un asterisco en la lista de especies. Se enlistan también seis taxa registrados en la literatura indicándolos con una referencia bibliográfica. A continuación del número de colecta se hace referencia al número de localidad según el mapa y la lista de localidades.

En la presente contribución se ha adoptado la nomenclatura de autores de monografías y revisiones taxonómicas recientes. El primer juego de ejemplares se depositó en el Herbario Nacional de México (MEXU).

1. Ladera del cerro Mono Pelón, cerca de la colonia El Diamante, municipio de Tecpatán, Chiapas. $17^{\circ} 18^{\prime}$ N-933'' W. Números recolectados: 1246-1249, 1519, 1523 , 1524, 1526, 1528, 1540-1542.

a. Selva alta o mediana perennifolia, sobre areniscas y lutitas del Oligoceno y Eoceno, entre los 400 y 1000 metros sobre el nivel del mar (msnm). En las partes bajas o planas la selva alta perennifolia está compuesta por Calophyllum brasiliense, Dialium guianense y Sickingia salvadorensis, entre otras. En las laderas de mayor inclinación a los 750 msnm aparecen Liquidambar y Talauma.

b. Los números 1493, 1494, 1497-1499 se colectaron a la orilla de un arroyo en Ran- 
cho Monte Armenio, Ejido Chintul, Tecpatán, Chiapas a unos $3 \mathrm{~km}$ al SE de la base del cerro Mono Pelón.

2. Rancho El Rosario, kilómetro 21.5 de la carretera a Francisco Rueda, municipio de Huimanguillo, Tabasco. 17050’N-933’'W. Números 1103.5, 1341 a-d. Encinar tropical, sobre suelos lateríticos derivados de sedimentos aluviales del Pleistoceno. Predomina Quercus oleoides acompañado de Guatteria amplifolia, Xylopia frutescens y Cocblospermum vitifolium, entre las plantas más importantes.

3. Colegio Superior de Agricultura Tropical, kilómetro 21.5 de la carretera Circuito del Golfo, Tabasco. 17059'N 933' 'W. Números 1061, 1132, 1163, 1348, 1349, 1360 1362. Selva alta o mediana subperennifolia de Bravaisia integerrima y Licania platypus, sobre suelos profundos y arcillosos.

4. Ciudad H. Cárdenas, Tabasco. 17059’N-93²3’W. Números 1363, 1364. Sobre pared húmeda. Números 1367, 1368. Cacaotal (Teobroma cacao), con sombra permanente de Erythrina americana, Samanea saman, Artocarpus altitlis, Guazuma ulmifolia y Bursera simaruba.

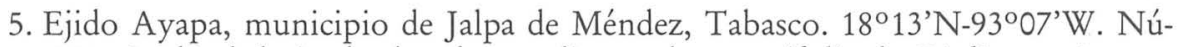
mero 1359. Acahual derivado de selva mediana subperennifolia de Dialium guianense y Scheelea liebmanii, sobre suelos lateríticos en afloramientos de depósitos pleistocénicos.

6. Selva de Dos Montes, atrás del aeropuerto, municipio del Centro, Tabasco. $18^{\circ} 00^{\prime}$ N-92 $49^{\prime}$ 'W. Número 1397. Selva mediana subperennifolia de Dialium guianense, Cynometra retusa y Scheelea liebmanii, sobre suelos relativamente profundos en lomeríos bajos de depósitos aluviales pleistocénicos.

7. Parque Museo de La Venta, Villahermosa, Tabasco. $18^{\circ} 00^{\prime} \mathrm{N}-92^{\circ} 56^{\prime} \mathrm{W}$. Números 1350,1351 . Selva muy perturbada con Tabebuia rosea, Sterculia apetala y Ceiba pentandra, entre otras especies arbóreas.

8. Municipio de Teapa, Tabasco, $1.5 \mathrm{~km}$ al W de Rancho Santa Fe. $17^{\circ} 38^{\prime}$ N-92 $59^{\circ}$ W. Números 1180,1181. Selva alta o mediana subperennifolia de Bravaisia integerrima y Licania platypus con Terminalia amazonia.

9. Centro Regional Tropical Puyacatengo, municipio de Teapa, Tabasco. 17031'N9255'W. Números 1352, 1476, 1479-1481. Selva alta perennifolia de Brosimum alicastrum, sobre suelos someros de calizas cársticas. Acompañan a B. alicastrum, Pouteria sapota, Dialium guianense y Sweetia panamensis, entre otras. Excepto por el número 1479, los ejemplares se colectaron en un cacaotal cerca de la selva.

10. Laderas del Cerro del Madrigal, municipio de Teapa, Tabasco. $17^{\circ} 32^{\prime} \mathrm{N}-92^{\circ} 55^{\prime} \mathrm{W}$. Números 1063, 1342. Selva alta perennifolia de Brosimum alicastrum.

11. Ejido Francisco Sarabia, segunda sección, municipio de Teapa, Tabasco. $17^{\circ} 37^{\prime} \mathrm{N}$ 9255’W. Números 1287-1289. Selva alta o mediana subperennifolia de Bravaisia intege- 
rrima. Los números 1287 y 1289 se colectaron en un cacaotal y en un pastizal, respectivamente.

12. Santa Rosa, municipio de Tacotalpa, Tabasco. $17^{\circ} 34^{\prime}$ N-92 $48^{\prime}$ W. Número 1064. Selva alta o mediana subperennifolia de Bravaisia integerrima.

13. Finca Villa Luz, Tapijulapa, municipio de Tacotalpa, Tabasco. $17^{\circ} 26^{\prime} \mathrm{N}-92^{\circ} 46^{\prime} \mathrm{W}$. Números 1062, 1345, 1424, 1433, 1434, 1444-1446. Restos de selva alta perennifolia con cultivo de café, sobre suelos profundos bien drenados. Se encuentran individuos de Pouteria sapota, Lonchocarpus hondurensis y Poulsenia armata, entre otros.

14. Cascadas de Agua Blanca, municipio de Mascuspana, Tabasco. $17^{\circ} 37^{\prime} \mathrm{N}-92^{\circ} 28^{\prime} \mathrm{W}$. Números 1457-1459, 1467, 1472, 1474. Selva alta perennifolia de Brosimum alicastrum y Dialium guianense, sobre suelos someros derivados de calizas cársticas. Acompañan a los dominantes Ceiba pentandra, Quararibea funebris y Pouteria campechiana, entre otras.

15. Municipio de Macuspana, Tabasco, $10 \mathrm{~km}$ al E de Colomo. $17^{\circ} 59^{\prime} \mathrm{N}-92^{\circ} 23^{\prime} \mathrm{W}$. Número 1065. Selva baja subperennifolia (bosque inundable de Haematoxylon campechianum), sobre suelos limoso-arcillosos de origen aluvial reciente.

16. Monte Grande, municipio de Jonuta, Tabasco. $17^{\circ} 57^{\prime} \mathrm{N}-92^{\circ} 17^{\prime} \mathrm{W}$. Números 1066 , 1223, 1343-1346. Selva mediana subperennifolia de Bucida buceras, sobre suelos inundables profundos de origen aluvial reciente. En el estrato arbóreo existen también Tabebuia rosea, Lonchocarpus hondurensis, y Ormosia macrocalix.

17. Municipio de Jonuta, Tabasco, $20.5 \mathrm{~km}$ al N de Zapatero. $17^{\circ} 53^{\prime} \mathrm{N}-92^{\circ} 09^{\prime} \mathrm{W}$. Número 1347. Acahual muy perturbado derivado de selva mediana subperennifolia de Bucida buceras, Tabebuia rosea, Albizzia longipedata y Spondias mombin están entre las especies comunes.

18. Municipio de Emiliano Zapata, Tabasco, $3.5 \mathrm{~km}$ al N de El Arenal. $17^{\circ} 41^{\prime} \mathrm{N}$ 913’ W. Números 1357, 1358. Acahual derivado de selva alta perennifolia de Terminalia amazonia y Vochysia hondurensis, sobre suelos arenosos y bien drenados.

19. Ejido Redención del Campesino, municipio de Tenosique, Tabasco. 17²0’ N911'W. Selva alta perennifolia de Terminalia amazonia:

a) Números 1308, 1317. Junto al río, sobre suelos profundos bien drenados. Acompañan a T. amazonia, Dialium guianense, Pouteria sapota y Poulsenia armata, entre otras.

b) Números 1320, 1321, 1353-1356. En laderas de rocas calizas en las que se encuentran Manilkara zapota, Calophyllum brasiliense y Dialium guianense.

\section{LISTA DE ESPECIES}

Anomobryum filiforme var. concinnatum (Spruce) Loesk. (Ochi, 1980 como Bryum filiforme var. concinnatum (Spruce) Boul.)

Barbula agraria Hedw. 1363 (Loc. 4). 
B. arcuata Griff. 1317 (Loc. 19a). En México, esta especie se conocía solamente de Nuevo León y Veracruz (Zander, 1981).

B. indica (Hook.) Spreng. 1181 (Loc. 8).

Brachymenium globosum Jaeg. Ochi (1980).

Bryohaplocladium microphyllum (Hedw.) Watanabe \& Iwats. 1065 (Loc. 15).

Bryum beyrichianum (Hornsch.) C.M. Ochi (1981).

B. capillare Hedw. 1289b, 1341c, 1353b, 1355 (Loc. 2, 11, 19b).

B. coronatum Schwaegr. 1364 (Loc. 4).

Callicostella pallida (Hornsch.) Aongstr. 1063a, 1163a, 1362, 1367b, 1434b; Welch (1966) (Loc. 3, 4, 10, 13).

Calymperes afzelii Sw. 1346, 1347a (Loc. 16, 17). Esta especie era mejor conocida como C. donnellii Aust. (cf. Reese, 1983).

Chryso-hypnum diminutioum (Hampe) Buck. 1320a, 1321 (Loc. 19b).

C. squarrosulum (Card.) 1474 Nishimurae And. (Loc. 14). Antes conocido solamente de Veracruz (Crum, 1951; Delgadillo, 1976) pero aparentemente también se encuentra en Puebla y San Luis Potosí.

Ectropothecium globitheca (C.M.) Mitt. 1498* (Loc. 1b).

Fissidens diplodus Mitt. 1344 (Loc.16).

F. zollingeri Mont. 1248*, 1287, 1361 (Loc. 1, 3, 11).

Funaria bygrometrica var. calvescens (Schwaegr.) Mont. 1246*, 1247* (Loc. 1).

Hypopterygium tamariscinum (Hedw.) Brid. 1526* (Loc.1).

Isopterygium diminutivum Bartr. 1342b, 1352a, 1359, 1360 (Loc. 3, 5, 9, 10).

Leucobryum albidum (P. Beauv.) Lindb. 1341b (Loc.2).

Meteoridium remotifolium (C.M.) Manuel (Manuel, 1977).

Neckeropsis disticha (Hedw.) Kindb. 1480b, 1481 (Loc. 9).

N. undulata (Hedw.) Reich. 1458a, 1480a (Loc. 9, 14).

Octoblepharum albidum Hedw. 1062, 1103.5b, 1223, 1341a, 1345, 1347c, 1354, 1444,

E. Rodríguez 5; Crum (1951); (Loc. 2, 13, 16, 17).

O. erectifolium Mitt. 1541* (Loc. 1).

Orthostichopsis tetragona (Hedw.) Broth. 1467 (Loc.14).

Papillaria nigrescens (Hedw.) Jaeg. 1446 (Loc.13).

Philonotis uncinata (Schwaegr.) Brid. 1368 (Loc. 4).

Pilotrichella rigida (C.M.) Besch. 1459a (Loc. 14).

Porotrichum longirostre (Hook.) Mitt. 1459b (Loc. 14).

Pseudocryphaea domingensis (Spreng.) Buck 1457 (Loc.14).

Sematophyllum caespitosum (Hedw.) Mitt. 1343, 1347b, 1348, 1350, 1351 (Loc. 3, 7, $16,17)$.

S. cuspidiferum Mitt. 1493* (Loc. 1b).

S. cf. sericifolium Mitt. 1523* (Loc. 1a).

S. subsimplex (Hedw.) Mitt. 1524* (Loc. 1a).

Stereophyllum radiculosum (Hook.) Mitt. 1358 (Loc.18).

Syrrhopodon incompletus Schwaegr. var. incompletus 1066, 1341d, 1397 (Loc. 2, 6, 16).

S. ligulatus Mont. 1103.5a (Loc. 2).

Taxipbyllum taxirameum (Mitt.) Fleisch. 1499* (Loc. 1b).

Taxithelium planum (Brid.) Mitt. 1061, 1063b, 1064b, 1163b, 1288, 1289a, 1320b, 1352b, 1356, 1357 (Loc. 3, 9, 10, 11, 12, 18, 19b). 
Thuidium delicatulum (Hedw.) Mitt. 1519* (Loc. 1a).

T. involvens 1434a, 1479 (Loc. 9, 13).

Tortula mniifolia (Sull.) Mitt. 1433 (Loc.13).

Trichosteleum subdemissum (Besch.) Jaeg. 1249, 1308, 1342a, 1353a, 1367a (Loc. 1, 4, 10, 19a, b).

Vesicularia poeppigiana (Hampe) Crum \& Steere 1064a, 1180 (Loc. 8, 12).

$V$. vesicularis 1132, 1349 (Loc. 3).

Zelometeorium patulum (Hedw.) Manuel 1445, 1459c, 1476 (Loc. 9, 13, 14).

En la lista anterior se citan 47 especies y variedades de musgos para la flora de Tabasco; sin embargo, en sentido estricto sólo podemos reconocer 38 taxa para ese estado pues las demás provienen de la zona limítrofe con Chiapas. Desde el punto de vista fitogeográfico no es posible efectuar un análisis de las afinidades de esta flora en tanto no se cuente con una mejor representación de los musgos. No obstante, se debe mencionar que, tomando en consideración la distribución de las especies en áreas circunvecinas, la flora de musgos de Tabasco parece ser una extensión de la de Veracruz y de la península de Yucatán. De los taxa citados en la lista anterior, 25 provienen de esta última área geográfica (cf. Delgadillo, 1984); la mayoría de ellos pertenece al elemento del Caribe que se distribuye de México al norte de América del Sur y es bien conocido en las Antillas. También es evidente que la flora incluye taxa típicamente tropicales (v.g., Callicostella pallida, Ectropothecium globitheca, Syrrhopodon ligulatus y Vesicularia poeppigiana) y otros de derivación templada como Bryohaplocladium microphyllum y Thuidium delicatulum. Estas consideraciones son predecibles dada la situación geográfica de Tabasco, pero el estudio global de su flora es necesario para completar las ideas sobre rutas de migración, grado de penetración de elementos tropicales al país y fluctuación altitudinal de los taxa desde las áreas vecinas.

AgRAdECIMIENTOS. Los autores agradecen la colaboración de Bernardina Bello y Ma. Ángeles Cárdenas en la obtención de datos sobre la flora de musgos de Tabasco. El mapa es obra de Felipe Villegas. Las colecciones se realizaron con el apoyo económico del Proyecto Flora Mesoamericana, Componente Tabasco.

\section{LITERATURA CITADA}

Crum, H.A., 1951. The Appalachian-Ozarkian element in the moss flora of Mexico with a check-list of all known Mexican mosses. Ph. D. Dissertation. Univ. Michigan, Ann Arbor.

Delgadillo M., C., 1976. Estudio botánico y ecológico de la región del Río Uxpanapa, Veracruz. No. 3. Los musgos. Publ. Inst. Invest. Rec. Bióticos 1 (2):19-28.

—- 1984. Mosses of the Yucatan Peninsula, Mexico. III Phytogeography. Bryologist 87:12-16.

Manuel, M.G., 1977. The genus Meteoridium (C. Müll.) Manuel, stat. nov. (Bryopsida: Meteoriaceae). Lind. bergia 4:45-55.

OchI, H., 1980. A revision of the Neotropical Bryoideae, Musci (First Part). J. Fac. Educ. Tottori Univ. 29:49-154.

_ 1981. A revision of the Neotropical Bryoideae, Musci (Second Part). J. Fac. Educ. Tottori Univ. 30:21-55.

REESE, W.D., 1983. American Calymperes and Syrrbopodon: identification key and summary of recent nomenclatural changes. Bryologist 86:23-30.

WELCH, W.H., 1966. The Hookeriaceae of Mexico. Bryologist 69:1-68.

ZANDER, R.H., 1981. Descriptions and illustrations of Barbula, Pseudocrossidium and Bryoerythrophyllum (p.p.) of Mexico. Cryptogamie, Bryol. Lichénol. 2:1-22. 